\title{
La transversalidad curricular desde las áreas de educación física y artística para una cultura ambiental
}

The curricular transversality from the areas of physical and artistic education for an environmental culture

\author{
Mario Sahamir Montes-Pacheco \\ mariosahamir0221@yahoo.es \\ Universidad Popular del Cesar, Valledupar \\ Colombia \\ https://orcid.org/0000-0002-4543-6882 \\ Iván Javier Movilla-de-Oro \\ movilladeportes@yahoo.es \\ Universidad Popular del Cesar, Valledupar \\ Colombia \\ https://orcid.org/0000-0003-1155-4546 \\ María-del-Carmen Jiménez-Barriosnuevo \\ mariacjimenez@unicesar.edu.co \\ Universidad Popular del Cesar, Valledupar \\ Colombia \\ https://orcid.org/0000-0002-2491-3731
}

Recibido: 03 de mayo de 2021

Aprobado: 04 de agosto de 2021 


\title{
RESUMEN
}

La creciente generación de residuos sólidos, que pone en evidencia el deterioro ambiental del planeta, hace necesario integrar el componente ambiental a los procesos formativos desde una visión transversal del currículo, si se aspira coadyuvar a una sociedad sostenible, donde las generaciones futuras puedan disfrutar un mundo mejor. Entonces, el objetivo se enmarco en realizar una revisión teórica sobre la transversalidad curricular como enfoque generador de Cultura Ambiental, para la reutilización de los residuos sólidos desde las áreas de educación física y artística, en el contexto de la Educación Ambiental. De allí que, metodológicamente, el artículo responde a una investigación teórico-descriptiva. Se concluye que la Educación Ambiental asumida curricularmente desde el principio de la transversalidad se constituye en vía que posibilita conocimientos y saberes interdisciplinarios, desde la integración de áreas como Educación Física y Artística, que coadyuvan a la construcción de una cultura ambiental para una sociedad sostenible.

Descriptores: Educación ambiental; educación física; educación artística; ambientalista. (Palabras tomadas del Tesauro UNESCO).

\begin{abstract}
The growing generation of solid waste, which highlights the environmental deterioration of the planet, makes it necessary to integrate the environmental component into the training processes from a transversal vision of the curriculum, if it is to contribute to a sustainable society, where future generations can enjoy a better world. Then, the objective was framed in carrying out a theoretical review on the curricular transversality as a generator approach of Environmental Culture, for the reuse of solid waste from the areas of physical and artistic education, in the context of Environmental Education. Hence, methodologically, the article responds to a theoretical-descriptive investigation. It is concluded that Environmental Education assumed curricularly from the beginning of transversality constitutes a path that enables interdisciplinary knowledge and knowledge, from the integration of areas such as Physical and Artistic Education, which contribute to the construction of an environmental culture for a sustainable society.
\end{abstract}

Descriptors: Environmental education; physical education; artistic education; environmentalists. (Words taken from the UNESCO Thesaurus). 


\section{CIENCIAMATRIA}

Revista Interdisciplinaria de Humanidades, Educación, Ciencia y Tecnología

Año VII. Vol. VII. N¹. Edición Especial. 2021

Hecho el depósito de ley: pp201602FA4721

ISSN-L: 2542-3029; ISSN: 2610-802X

Universidad Nacional Experimental Francisco de Miranda (UNEFM). Santa Ana de Coro. Venezuela

Mario Sahamir Montes-Pacheco; Iván Javier Movilla-de-Oro; María-del-Carmen Jiménez-Barriosnuevo

\section{INTRODUCCIÓN}

El medio ambiente se sitúa en el escenario mismo de la vida que entrelaza los sistemas naturales y los sistemas sociales (Saldaña y Mesina, 2014; p.4). Es por ello que durante muchos años uno de los grandes retos del ser humano ha sido poder desarrollarse y llegar a un nivel de avance, sin menoscabo del resto de lo existente en la tierra; es decir, lograr progresar sin repercutir en el ambiente, pues el desarrollo de la vida humana tiene sustento en la naturaleza. No obstante, todo esto simplemente ha quedado en un anhelo, dado que, inminentemente la acción humana ha impactado de manera directa y masiva en el deterioro del planeta. Pues, la actividad del hombre cada día se hace más agresiva, destruyéndose con ello la fuerza ambiental del planeta (CEUPE, s/f).

Si bien es cierto que existen muchos factores que ineludiblemente perjudican o dañan el entorno ambiental, hay uno que durante mucho tiempo ha venido creciendo de manera exponencial y es la generación de los residuos sólidos. En tal sentido, este problema de desechos sólidos genera un panorama mundial nada alentador a menos que se tomen medidas urgentes, pues se prevé que los desechos a nivel mundial aumenten a 3400 millones de toneladas para el 2050 , es decir, en un $70 \%$ con respecto a los niveles actuales (Banco Mundial [BM], 2018). Sin embargo, es pertinente resaltar que existen muchos residuos sólidos a los que se les puede aplicar procesos de selección, reciclaje y reutilización, considerando que, para poder actuar de forma correcta frente a su manejo, se debe tener un adecuado conocimiento, tornándose los procesos educativos preponderante para ello.

Contextualizando lo planteado al ámbito colombiano, se tiene que, para el 2018 la producción de residuos sólidos y productos residuales anual ascendió a más de 24 millones de toneladas, de las cuales, el $48,8 \%$ (12,1millones de toneladas) corresponde a la tasa de aprovechamiento (Departamento Administrativo Nacional de Estadística [DANE], 2018). 


\section{CIENCIAMATRIA}

Revista Interdisciplinaria de Humanidades, Educación, Ciencia y Tecnología

Año VII. Vol. VII. N¹. Edición Especial. 2021

Hecho el depósito de ley: pp201602FA4721

ISSN-L: 2542-3029; ISSN: 2610-802X

Universidad Nacional Experimental Francisco de Miranda (UNEFM). Santa Ana de Coro. Venezuela

Mario Sahamir Montes-Pacheco; Iván Javier Movilla-de-Oro; María-del-Carmen Jiménez-Barriosnuevo

Los residuos sólidos generados en el subsector educativo dependen del tipo y el tamaño de la institución (Área Metropolitana del Valle de Aburrá, 2008). Asimismo, se puede evidenciar, en un sinnúmero de instituciones educativas, la inadecuada disposición final de residuos sólidos. Situación que se refleja en la acumulación de empaques de alimentos y bebidas, restos de papel y cartón, entre otros, arrojados en lugares no dispuestos para tal fin, dejando a un lado actividades de reutilización y reciclaje, a pesar de la existencia de canecas de basura. De igual forma, los residuos orgánicos de hojas secas tienen un inadecuado manejo, se realizan quemas a cielo abierto, generándose un impacto negativo en el ambiente causando afectación a la comunidad educativa en general. Toda esta realidad se refleja en los ambientes físicos escolares, aquejando ineludiblemente los procesos educativos.

Atendiendo a esto, es vital que se desarrollen valores ecológicos, relacionados con la responsabilidad y el respeto hacia la naturaleza. Por consiguiente, se hace necesario integrar el componente ambiental a los procesos formativos y de aprendizaje desde la transversalidad de todas las áreas del currículo, considerando que, más allá de un aprendizaje sobre contenidos para el manejo de los residuos sólidos, es necesario formar a los estudiantes alrededor de la importancia que tiene el manejo de los residuos sólidos para la conservación y sostenibilidad de los ecosistemas. Convirtiéndose esto, en una responsabilidad inherente a las diferentes instituciones educativas del país.

Con base en lo planteado, es menester cuestionar ¿Cómo la transversalidad curricular se constituye en un enfoque para la generación de una cultura ambiental (CA), desde las áreas de educación física y artística y en el marco de la Educación Ambiental (EA)? De allí que, emerge la necesidad de hacer una revisión de la producción académica-científica a fin de abordar el principio de la transversalidad curricular como enfoque generador de una CA, para la reutilización de los residuos sólidos desde las áreas de educación física y artística, en el contexto de la EA. 
Metodológicamente, el presente artículo de revisión refiere a un estudio de corte teóricodescriptivo, devenido de un sistemático trabajo de investigación bibliográfica. En tal sentido, se asumió como lógica de acción el despliegue de los procesos de: rastreo, identificación, organización, sistematización, análisis e interpretación del conjunto de datos e informaciones (Sanchez, 2011), a fin de responder el cuestionamiento que se hace, el cual, es guiador del proceso investigativo (Mera, Barreiro, Bermúdez, Cedeño y Vera, 2020).

La búsqueda y análisis de los documentos relacionados con el objeto de estudio se dio bajo los criterios de que estos fueran producciones académicas-científicas como artículos de revistas, conferencias y publicaciones institucionales, de tipo electrónicos, en idioma español. Asimismo, se establecieron tres subtemas denominados: la transversalidad del currículo, cultura ambiental y gestión de residuos sólidos, como categorías de análisis que guiaron la búsqueda de información (Sánchez, 2011). Estas categorías como macro descriptores se dispusieron de distintas formas en el proceso de rastreo, a propósito de ampliar los ámbitos de exploración.

Para cerrar, el proceso de análisis y síntesis de la información considerada, se ha organizado en tres apartados. En tal sentido, el primero refiere a la cultura y la EA como sendas para la construcción de una sociedad inmersa en el desarrollo sostenible (DS); el segundo, describe la transversalidad curricular como enfoque pedagógico para la generación de CA y, el ultimo, hace precisiones sobre la reutilización de residuos sólidos como propuesta de EA integradora de las áreas de Educación Física y Educación Artística. 


\section{DESARROLLO}

\section{La cultura y la educación ambiental como sendas para la construcción de una sociedad inmersa en el desarrollo sostenible}

A nivel mundial, se ha incrementado la preocupación por la problemática medioambiental, dado el eminente y desmedido deterioro de los ecosistemas, la sobreexplotación de los recursos y el inadecuado uso de los espacios naturales Espinoza (2007). Estas situaciones, que son de gran complejidad, han causado grandes afectaciones a nivel de la salud; en la producción de alimentos y materia prima; del agua; a la vez que han acrecentado la incidencia del cambio climático y los desastres naturales en el planeta. De allí que es menester, como expresa Ezquerra (2020), que el hombre asuma como prioridad el desarrollo de una sociedad sostenible.

En este contexto y como forma de generar posibles soluciones a los cambios que impactan el medio ambiente emerge el DS; el cual, conceptualmente refiere al resultado de una acción concertada de las naciones para impulsar un modelo de desarrollo económico mundial acorde con la preservación del medio ambiente y la igualdad social (Universidad de Nuevo León, s/f). Esto, en términos de Espinoza (2007), representa un "reconocimiento a la necesidad de compatibilizar el continuo crecimiento económico con la equidad social y con la protección y administración eficaz y eficiente del medio ambiente" (p.17).

Concretamente el DS, que "hunde sus raíces en la crítica al desarrollo económico en general ante los altos niveles de degradación del medio ambiente" (Alaña, Capa y Sotomayor, 2017, p.94), emerge como fundamento rector para el progreso de las sociedades a largo plazo (Asamblea General de las Naciones Unidas, 2010), a fin de asegurar la satisfacción de las necesidades que los ciudadanos tienen en el presente, sin menoscabo de la capacidad de las futuras generaciones para satisfacer sus propias necesidades (Asamblea General de las Naciones Unidas, 1987). De allí que, el marco legal de Colombia lo define como toda acción que "conduce al crecimiento económico, al 


\section{CIENCIAMATRIA}

Revista Interdisciplinaria de Humanidades, Educación, Ciencia y Tecnología

Año VII. Vol. VII. N¹. Edición Especial. 2021

Hecho el depósito de ley: pp201602FA4721

ISSN-L: 2542-3029; ISSN: 2610-802X

Universidad Nacional Experimental Francisco de Miranda (UNEFM). Santa Ana de Coro. Venezuela

Mario Sahamir Montes-Pacheco; Iván Javier Movilla-de-Oro; María-del-Carmen Jiménez-Barriosnuevo

mejoramiento de la calidad de la vida y al bienestar social, sin agotar la base de recursos naturales renovables en que se sustenta, ni deteriorar el medio ambiente o el derecho de las generaciones futuras a utilizarlo en satisfacción de sus propias necesidades" (Ley 99, 1993).

Asumido así el DS, las sociedades han de buscar las maneras para que la actividad económica se despliegue de manera amigable y armoniosa con el sistema natural y permita un mejoramiento de la calidad de vida de la especie humana. Por consiguiente, deben ejecutar prácticas enmarcadas en el aprovechamiento de los recursos disponibles de manera eficiente, la promoción de la cultura del reciclaje, la reutilización y uso de tecnologías limpias, que coadyuven a restaurar los ecosistemas que han sufrido daños, con la finalidad de promover la autosuficiencia regional, reconociendo el rol imprescindible de la naturaleza para el bienestar de los seres humanos (Colombia Aprende, 2019).

Aquí, cabe acotar lo expresado por Ezquerra (2020), en relación a que es menester asumir algunas propuestas básicas para construir una sociedad sostenible, si se quiere que las generaciones futuras puedan disfrutar de un mundo mejor. En tal sentido, algunos de estas proposiciones expuestas por la propia Ezquerra, son:

- Mejorar de la calidad de vida de la especie humana, en el marco de los derechos universales.

- Implementar la cultura del cuidado y respeto a todos los seres humanos y especies que habitan el planeta Tierra.

- Reducir del consumo innecesario de los recursos naturales no renovables y aprovechar de manera eficiente los renovables.

- Desarrollar el reciclaje de materias primas, materiales y equipos.

- Informar y educar a la sociedad para la conservación e integración de un mundo sostenible, mediante políticas educativas, económicas y sociales adaptadas a las necesidades de los nuevos tiempos que están por venir. 


\section{CIENCIAMATRIA}

Revista Interdisciplinaria de Humanidades, Educación, Ciencia y Tecnología

Año VII. Vol. VII. N¹. Edición Especial. 2021

Hecho el depósito de ley: pp201602FA4721

ISSN-L: 2542-3029; ISSN: 2610-802X

Universidad Nacional Experimental Francisco de Miranda (UNEFM). Santa Ana de Coro. Venezuela

Mario Sahamir Montes-Pacheco; Iván Javier Movilla-de-Oro; María-del-Carmen Jiménez-Barriosnuevo

- Crear alianzas entre los distintos países a nivel mundial para tratar el tema medioambiental.

Considerando lo anterior, la construcción de una sociedad inmersa en el desarrollo sostenible donde lo económico, los social y lo natural se ha de constituir en un sistema armónico, va más allá de la realización de actividades para la protección del entorno, ya que, implica un cambio de pensamiento y actitud ante las formas de vida y sobre las políticas que regulan dicha protección del medio ambiente.

Ahora bien, para la consecución de este principio de desarrollo sostenible, las estrategias que se crean están directamente ligadas al concepto de CA y de EA. En tal sentido, es importante hacer algunas disquisiciones en torno a estas dos categorías conceptuales. Antes que nada, se debe entender la cultura "como esa totalidad compleja" (Taylor, 1871 referenciado por Lévi-Strauss, 1974), que implica conocimientos, ideas, pensamientos, formas de comportamientos, actitudes, técnicas, instrumentos, prácticas, costumbres, creencias, instituciones y todas las demás capacidades y hábitos adquiridos, por medio de la cual, cada grupo humano trata de ajustarse a su ambiente (Ortiz, 2008 en García, 2015). Significa entonces, como expresa Bayón (2006), que "la cultura establece los parámetros de relación y reproducción social con relación a la naturaleza" (p.91).

Lo planteado, conlleva a considerar que la cultura se funda en la relación del hombre con su entorno, pues, toda sociedad humana es un fruto entrelazado de la evolución natural y del desarrollo social. Lo que significa, que el concepto de cultura esta inextricablemente unido al concepto de ambiente. Tanto así, que la identidad y los procesos culturales de cada sociedad están influenciados por el entorno natural en el que se desarrollan y, de manera recíproca, cada civilización ineludiblemente deja una huella en los entornos naturales donde se encuentran, siendo el resultado de estos procesos determinantes del estado del entorno socioambiental (García, 2015). 


\section{CIENCIAMATRIA}

Revista Interdisciplinaria de Humanidades, Educación, Ciencia y Tecnología

Año VII. Vol. VII. N¹. Edición Especial. 2021

Hecho el depósito de ley: pp201602FA4721

ISSN-L: 2542-3029; ISSN: 2610-802X

Universidad Nacional Experimental Francisco de Miranda (UNEFM). Santa Ana de Coro. Venezuela

Mario Sahamir Montes-Pacheco; Iván Javier Movilla-de-Oro; María-del-Carmen Jiménez-Barriosnuevo

Ahora bien, el estado del entorno tanto social como natural, que actualmente se muestran en franco y progresivo deterioro, deviene de los modelos de pensamiento, y creencias que el hombre ha establecido y muestra con sus modos de ser, actuar y comportarse. Por consiguiente, es vital el desarrollo de una cultura ambiental adecuada, en el marco de la formación humana y ciudadana desde las primeras etapas educativa. En este contexto de crisis ambiental que le sobreviene al planeta, la "EA constituye una plataforma para desarrollar la cultura ambiental" (García, 2015: p.84), y un instrumento para atender dicha emergencia, haciendo que el hombre se replantee la manera de interactuar con el medio ambiente, ahora enfocado en el DS.

Históricamente el auge de la EA se puede situar en la década de los 70, como forma de concientizar la situación del deterioro ambiental del planeta y, aunque inicialmente solo conto con la participación de organizaciones no gubernamentales y de la educación no formal, progresivamente se fueron incorporando iniciativas y acuerdos en el ámbito institucional y de la educación formal, con la introducción de paquetes didácticos y unidades de aprendizaje aislados. No obstante, hoy día la EA se constituye en un programa estratégico que se despliega en la mayoría de los sistemas educativos a nivel mundial (Fuentes, Caldera, y Mendoza, 2006).

Considerando lo planteado, es importante puntualizar algunas nociones de diversos autores sobre EA, teniendo en cuenta que dicho concepto es dinámico, ya que, se desenvuelve a la par como lo hace el discernimiento y la idea de medio ambiente que tiene el hombre. En tal sentido, Roque (2003, referenciado por García, 2015) asume a la EA como un proceso que permite comunicar e internalizar la cultura ambiental; por tanto, debe reflejar un enfoque integrado que apunte al desarrollo sostenible. Asimismo, para Calderón y Caicedo (2019), la EA es un proceso de tipo epistémico, ético o cosmológico, la cual, se constituye en elemento integral de la política actual que permite la consolidación de valores supremos como la igualdad, la fraternidad, la libertad, la justicia y la dignidad. 


\section{CIENCIAMATRIA}

Revista Interdisciplinaria de Humanidades, Educación, Ciencia y Tecnología

Año VII. Vol. VII. N¹. Edición Especial. 2021

Hecho el depósito de ley: pp201602FA4721

ISSN-L: 2542-3029; ISSN: 2610-802X

Universidad Nacional Experimental Francisco de Miranda (UNEFM). Santa Ana de Coro. Venezuela

Mario Sahamir Montes-Pacheco; Iván Javier Movilla-de-Oro; María-del-Carmen Jiménez-Barriosnuevo

Avendaño (2012), sostiene que la EA ha de ser asumida como un proceso educativo integral y continuo para la generación de experiencias y saberes útiles sobre la naturaleza y su conservación ecológica. A la vez, Marazek (1996, citado por Flores, 2012), la define como el proceso interdisciplinario, con la cual, se puede construir un nuevo tipo de conciencia denominada planetaria (Morín, 2004). No obstante, para ello, se requiere que el hombre, de manera individual y colectiva, logre comprender la naturaleza compleja del entorno natural y social; a la vez que adquiera conocimientos, valores, comportamientos y habilidades prácticas para participar de manera responsable y competente en la prevención y la gestión de los problemas y la calidad ambiental (Caduto, 1992 en Martínez, 2010).

Todas estas nociones precisadas, develan que la EA es un proceso epistémico, éticoaxiológico y político que tiene como eje a la CA focalizada en el DS. Asimismo, ha de consolidarse, desde los principios de integralidad y continuidad, mediante experiencias generadoras de aprendizajes y saberes interdisciplinarios, para la creación de conciencia planetaria, a partir de la comprensión y la acción transformadora de la realidad del entorno socioambiental. Esto último, de develar la EA como acción transformadora, se concatena con lo expresado por Paz, Avendaño y Parada (2014), en el sentido de que busca responder a los inminentes cambios socioestructurales que la sociedad demanda hoy dia.

En otro orden de ideas, se destaca que la fundamentación de la EA se encuentra tanto en las normas internacionales como locales $y$, aunque su desarrollo conceptual ha sido amplio y complejo, su utilización es imprescindible dentro de los procesos educativos formales e informales (Paz, et al., 2014); pues, se busca formar un ser humano activo y responsable en la construcción de una manera distinta de relación entre el hombre y la naturaleza. En donde el sentir humano y los valores estéticos y ético-morales sean más diáfanos, aunado, a que las habilidades prácticas y la labor científica investigativa se hagan más eficiente en dirección de la CA (Pesantez, 2014). 
Se puede afirmar entonces que, la CA y la EA buscan fortalecer la conciencia de las personas, utilizando procesos educativos formales e informales, tratando de crear condiciones para establecer una relación armónica entre la naturaleza y las actividades que realizan las personas, Todo, con la finalidad de alcanzar el desarrollo sostenible y, por ende, garantizar la calidad de vida de las actuales y futuras generaciones (Pesantez, 2014). Sin embargo, equilibrar las relaciones individuo-sociedad-ambiente, además de entender profundamente las problemáticas en la dimensión ambiental, requiere reconstruir lo existente a partir de vincular las dimensiones socio-cultural, tecnoeconómica y político-institucional con las dimensiones del ser humano, que interactúa con su entorno (Ardila, Vergara y González, 2018).

De allí que se hace necesario hacer un cambio en los valores relacionados entorno; puesto que, en la actualidad se otorga mayor importancia al crecimiento económico que a la protección del hábitat, siendo que, esta última es generadora de grandes aportes a los seres humanos. Por ello, es fundamental la implementación de modelos educativos y curriculares innovadores enmarcados en la CA, para que, tanto docentes y estudiantes, como grupos sociales comunitarios tomen un papel activo dentro del proceso de EA, con el propósito de generar mayor conciencia y conocimientos significativos sobre cómo generar y preservar una relación armoniosa con el entorno y en pro del DS.

\section{La transversalidad curricular como enfoque pedagógico para la generación de cultura ambiental}

El progresivo deterioro del entorno ambiental se ha convertido en una problemática mundial de interés alarmante. De allí que, organismos internacionales como la UNESCO, el Proyecto de Naciones Unidas para el Desarrollo (PNUD) y el Proyecto de Naciones Unidas para el Medio Ambiente PNUMA, han propuesto directrices para la inclusión del componente ambiental como parte de las políticas educativas, a fin de promover, desde 
el ámbito pedagógico y sobre todo con los más pequeños, el desarrollo y fortalecimiento de una CA (Fuentes, Caldera, y Mendoza, 2006).

Considerando lo anterior, es importante traer a colación a Esquivel, Cifuentes, Suarez y Delgado (2018), cuando afirma que el ser humano como ser social, necesita de espacios que le permitan relacionarse con las demás personas, sin embargo, por causa de estas interacciones se ven afectados los entorno. De allí que es vital que la EA se vuelva un instrumento de las Instituciones Educativas, para enseñar y aprender que la naturaleza es algo inherente al ser humano, puesto que hace parte de la cotidianidad.

Así, la EA se muestra como una herramienta fundamental que permite a los individuos desarrollar una cultura enfocada en la sostenibilidad ambiental y la preservación de su propio entorno y, con ello, realizar cambios axiológicos, conductuales, de estilos de vida, a la vez, que amplíen los conocimientos y saberes con la finalidad de impulsar acciones para la prevención y mitigación de las problemáticas actuales y futuras (Severiche, Gómez y Jaimes, 2016).

Ahora bien, materializar la EA en el contexto educativo implica superar la idea de que es una disciplina independiente que se ha de asumir como asignatura aislada en los currículos; por lo contrario, como describe Tobasura y Sepúlveda (1997, referenciados por Fuentes, Caldera y Mendoza, 2006), es menester considerarla desde una visión sistémica e integradora de todas las disciplinas y saberes. Esta perspectiva hace que la EA se constituya en un proceso interdisciplinario que elimina las fronteras entre las disciplinas, posibilitando una forma de pensar y proceder que permite, al conocer la realidad objetiva, poder resolver cualquiera de los complejos problemas que esta plantea Casaña (2017).

Poniendo en contexto lo planteado, se tienen que el gobierno colombiano ha instaurado directrices específicas que se recogen en una Política Nacional de Educación Ambiental donde se plasma la intencionalidad de promover, desde el marco de una cultura ética y responsable del manejo sostenible del ambiente, una educación con visión de formación 


\section{CIENCIAMATRIA}

Revista Interdisciplinaria de Humanidades, Educación, Ciencia y Tecnología

Año VII. Vol. VII. N¹. Edición Especial. 2021

Hecho el depósito de ley: pp201602FA4721

ISSN-L: 2542-3029; ISSN: 2610-802X

Universidad Nacional Experimental Francisco de Miranda (UNEFM). Santa Ana de Coro. Venezuela

Mario Sahamir Montes-Pacheco; Iván Javier Movilla-de-Oro; María-del-Carmen Jiménez-Barriosnuevo

integral del ser humano, para que este haga lectura sistémica de la realidad ambiental e incidir conscientemente en su transformación de sus problemas (Ministerio del Ambiente, Vivienda y Desarrollo Territorial [MAVDT] y Ministerio de Educación Nacional [MEN], 2003).

En dicha política se específica, además, que las situaciones ambientales son enmarañadas dado el cumulo de interacciones que suceden en el sistema socioambiental. En tal sentido, su abordaje debe darse desde una perspectiva de trabajo interdisciplinario de permanente análisis y síntesis, puesto que, se considera que no es posible encontrar toda la información o metodologías necesarias para abordar dichas problemáticas, en una sola área o asignatura.

Concretamente, para comprender los complejos fenómenos ambientales, es necesario buscar soluciones de manera conjunta. Por consiguiente, se le tiene que dar cabida a la participación de diversas perspectivas y distintos ámbitos del conocimiento; y, con la integración de los distintos argumentos, se logre una comprensión y explicación global del fenómeno. Considerando esto, la transversalidad de la EA en el currículo se convierte en un elemento clave para que las directrices ofrecidas por los entes gubernamentales puedan ejecutarse de manera satisfactoria.

Es de saber que etimológicamente el adjetivo transversal es un término que proviene del latín y está conformado por el prefijo trans que significa "de un lado a otro", el vocablo versus que puede traducirse como "dado vueltas" y el sufijo al que equivale a "relativo a". Por tanto, puede referir a lo que se ubica atravesado de un extremo hacia otro; lo que se desvía de la normal línea recta y/o a lo que se interpone de manera perpendicular con aquello de que se trata (Diccionario etimológico deChile.net)

En cuanto a la transversalidad en el ámbito del currículo educativo, Aparicio y Rodríguez (2019) y Ferrini (1997) explican que este es un paradigma que se constituye con la intencionalidad de generar una formación integral y centrada en valores, tratando de superar el enfoque temático de los contenidos curriculares que permean el aprendizaje, 


\section{CIENCIAMATRIA}

Revista Interdisciplinaria de Humanidades, Educación, Ciencia y Tecnología

Año VII. Vol. VII. N¹. Edición Especial. 2021

Hecho el depósito de ley: pp201602FA4721

ISSN-L: 2542-3029; ISSN: 2610-802X

Universidad Nacional Experimental Francisco de Miranda (UNEFM). Santa Ana de Coro. Venezuela

Mario Sahamir Montes-Pacheco; Iván Javier Movilla-de-Oro; María-del-Carmen Jiménez-Barriosnuevo

para asumir uno más holístico e integrativo. En tal sentido, y como forma de entender mejor la idea de transversalidad, se presentan los siguientes supuestos a partir de lo que expone la propia Ferrini:

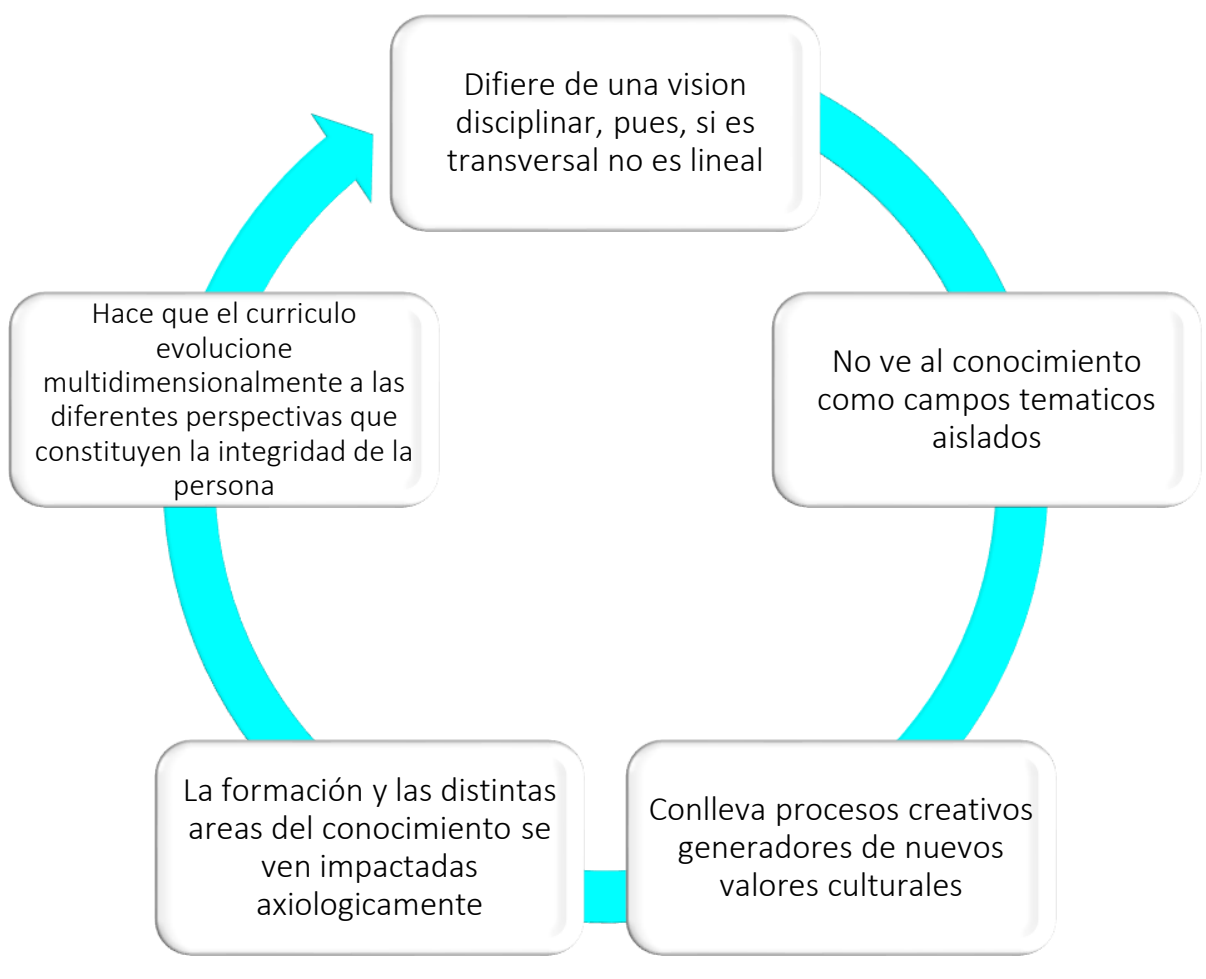

Figura 1. Supuestos de la Transversalidad.

Fuente: Adaptado de Ferrini (1997). 
Así mismo, Henríquez y Reyes explican textualmente que la transversalidad refiere a: ...contenidos culturales relevantes y valiosos, necesarios para la vida y la convivencia, que dan respuesta a problemas sociales y que contribuyen a formar de manera especial el modelo de ciudadano que demanda la sociedad, porque a través de una educación en valores los alumnos se sensibilizan, toman posiciones ante dichos problemas, emiten juicios críticos y actúan con un compromiso libremente asumido. Esto significa que son temas que no necesariamente tienen que conformar una asignatura en particular ni recibir un tratamiento especial dentro del currículo, sino que deben abordarse en todas las áreas que lo integran y en toda situación concreta de aprendizaje" (Henríquez \& Reyes, 2008; p.14).

Cabe resaltar que este paradigma de la transversalidad se forja a partir de temas y/o ejes transversales, que se tornan esenciales para una práctica pedagógica innovadora. Pues, estos refieren a problemas y conflictos actuales, que devienen de distintas esferas y dimensiones humanas, por los cuales, hay que tomar posición personal y colectiva, para su abordaje (Argueta, 2009). Además, como enuncia Ferrini (1997), "responden al mundo de la vida desde el momento que parte de la vida cotidiana" (p.6); dado que, se establecen a partir del análisis que se hace de la realidad social y en virtud de las situaciones problemáticas no resueltas causadas por el modelo de desarrollo actual (Palos, 2000 referenciado por Aparicio, Rodríguez, Beltrán y Sampedro, 2014).

La distinción entre temas y ejes transversales radica, principalmente, en que el primero son temas sociales emergentes que se abordan como contenidos educativos no disciplinares; mientas que el segundo, se constituyen en macro líneas ordenadoras y estructuradores de los temas sociales emergentes que permean el currículo (Aparicio y Rodríguez, 2019).

Concretamente, la incorporación de la educación ambiental como elemento transversal en el currículo, se focaliza en la necesidad de generar educandos con una actitud crítica ante las actuales formas de vida cotidiana y con capacidad para observar, entender y proyectarse en las problemáticas socioambiental que se generan en la realidad, a fin de 


\section{CIENCIAMATRIA}

Revista Interdisciplinaria de Humanidades, Educación, Ciencia y Tecnología

Año VII. Vol. VII. N¹. Edición Especial. 2021

Hecho el depósito de ley: pp201602FA4721

ISSN-L: 2542-3029; ISSN: 2610-802X

Universidad Nacional Experimental Francisco de Miranda (UNEFM). Santa Ana de Coro. Venezuela

Mario Sahamir Montes-Pacheco; Iván Javier Movilla-de-Oro; María-del-Carmen Jiménez-Barriosnuevo

coadyuvar en la construcción de una sociedad inmersa en la conjunción sistémica y equilibrada de lo económico, los social y lo natural, para el desarrollo sostenible.

En este ámbito y para transversalizar todo el currículo existen múltiples metodologías. Empero, entre estas variadas formas, es importante desplegar un proceso asumiendo los temas ambientales como estructuras epistémicas y del conocimiento para ser abordados en las distintas áreas curriculares, desde una postura práctica e interdisciplinar. Decisión que le da contexto a las áreas curriculares como espacios de aprendizaje, al interrelacionarlas con esferas de la realidad problematizada; además, de dotarlas de valor funcional o de aplicación inmediata, para la comprensión y posible transformación de los problemas que se configuran en la realidad misma (Severiche, Gómez y Jaimes, 2016). Aquí, cabe agregar que los proyectos ambientales se establecen como la estrategia idónea para desarrollar, de manera transversal en el currículo, los programas de EA y, con los cuales, se puede lograr la construcción de un conocimiento interdisciplinar, a propósito de forjar, en los estudiantes y ciudadanos en general, una CA que le permita adecuarse de manera equilibrada al medio ambiente y con ello se propicie el DS.

Muestra de ello, son los Proyectos Ambientales Escolares (PRAE), que surgen como expresa Restrepo y Restrepo (2017), por la necesidad de implementar, en las escuelas y colegios de Colombia, una EA que facilite la incorporación del conocimiento y el cuidado del ambiente. Es de saber que históricamente, los PRAE se establecen mediante el decreto 1743 de 1994; asimismo, se crean como uno de los ejes transversales del currículo de todos los niveles de la educación formal y con base en concebir la EA como estrategia interdisciplinaria para investigar y tratar los problemas ambientales desde una visión sistémica del entorno, a través de proyectos escolares pero con proyección comunitaria y desde una visión sistémica del medio ambiente (MAVDT/MEN, 2003). Considerando lo expresado por el MinEducación en publicación de Altablero (2005), los PRAE se pueden definir como propuestas pedagógicas que, desde una visión de la formación para el desarrollo sostenible, están centradas en estudiar y comprender los 
problemas y las potencialidades ambientales, a nivel local, regional y nacional. En tal sentido, se constituyen en eje articulador de saberes y espacios de participación y reflexión. no solo de estudiantes, sino de toda la comunidad escolar, para la construcción de soluciones acordes con las dinámicas naturales y socioculturales; es decir, en pro de una gestión sostenible del entorno de manera multidimensional.

\section{La reutilización de residuos sólidos como propuesta transversal para la Generación de Cultura Ambiental desde las Áreas de Educación Física y Artística}

En el marco de la gestión ambiental, asumida como proceso que está orientado a resolver, mitigar y/o prevenir los problemas de carácter ambiental, con el propósito de lograr un DS (Red de Desarrollo Sostenible de Colombia, s/f), el manejo de los residuos sólidos (RS) ocupa un lugar preponderante. Contextualizando esta idea al ámbito educativo, y considerando que las instituciones escolares se convierten en actores importantes en la generación de RS (Jibril et al., 2012 citados por Choles, 2013), se tiene que la reutilización de los RS como propuesta educativa, concebida desde el principio de la transversalidad, se constituye en un espacio idóneo para la generación de CA, a partir de la integración de diversas áreas de aprendizaje como la Educación Física y la Educación Artística.

A propósito de lo planteado, es importante destacar que los RS son todos los materiales producto de las actividades domésticas, comerciales, industriales, agrícolas, mineras y municipales, los cuales, pueden presentarse en forma sólida, liquida o contenido en forma gaseosa exceptuando las aguas residuales, que requiere ser eliminado de alguna forma (Romero, 2017). Asimismo, su manejo implica desplegar un conjunto de operaciones y prácticas, comunitarias y políticas, para darles una adecuada disposición, con el fin de evitar y/o minimizar la generación de residuos e incrementar el aprovechamiento de éstos (Área Metropolitana del Valle de Aburrá, 2008). 


\section{CIENCIAMATRIA}

Revista Interdisciplinaria de Humanidades, Educación, Ciencia y Tecnología

Año VII. Vol. VII. N¹. Edición Especial. 2021

Hecho el depósito de ley: pp201602FA4721

ISSN-L: 2542-3029; ISSN: 2610-802X

Universidad Nacional Experimental Francisco de Miranda (UNEFM). Santa Ana de Coro. Venezuela

Mario Sahamir Montes-Pacheco; Iván Javier Movilla-de-Oro; María-del-Carmen Jiménez-Barriosnuevo

En este contexto del manejo de los RS, se tiene que saber diferenciar entre reciclaje y reutilización de RS. En tal sentido, la reutilización es el proceso que se le aplica a cualquier residuo sólido para darle un nuevo uso, ya sea con el mismo u otro fin para el que fueron creados, generalmente en el mismo ámbito doméstico; mientras que, al reciclar los residuos, los materiales de los que están hechos se transforman en otros objetos, pero, por medio de un proceso industrial.

Considerando lo anterior, la reutilización se presenta como proceso clave para el manejo de los RS dentro de las instituciones educativas; pues, con ello se contribuye a la creación de cultura ambiental, a la vez que se suma fuerza a la conservación del ambiente los recursos naturales y la energía, con la reducción de la cantidad de basura producto de las distintas materias primas que puede requerir cualquier tipo de institución educativa para su proceso formativo.

Asimismo, se constituye en una estrategia pedagógica que posibilita la creación de "conciencia para la conservación, protección y mejoramiento del medio ambiente, de la calidad de la vida, del uso racional de los recursos naturales, de la prevención de desastres, dentro de una cultura ecológica y del riesgo y de la defensa del patrimonio cultural de la nación", tal como reza el artículo 5 de la Ley 115 (1994).

Es por ello que, parafraseando a Osorio (2012 en Garrido, 2016), se puede decir que la reutilización, en el contexto educativo, es y debe asumirse como una estrategia didáctica que permite la construcción de espacios de aprendizajes, a través de la ejecución de actividades enmarcadas en recuperar y transformar diferentes materiales, a la vez que se busca reducir el volumen de desperdicios y residuos sólidos.

En este sentido, tomar un elemento que para algunos es un desecho y convertirlo en un material didáctico, genera un impacto positivo tanto en el proceso de aprendizaje como en el ámbito de la sostenibilidad. Así, la reutilización se constituye en instrumento para la enseñanza y el aprendizaje de habilidades físicas y artísticas dentro de la escuela, con 


\section{CIENCIAMATRIA}

Revista Interdisciplinaria de Humanidades, Educación, Ciencia y Tecnología

Año VII. Vol. VII. N¹. Edición Especial. 2021

Hecho el depósito de ley: pp201602FA4721

ISSN-L: 2542-3029; ISSN: 2610-802X

Universidad Nacional Experimental Francisco de Miranda (UNEFM). Santa Ana de Coro. Venezuela

Mario Sahamir Montes-Pacheco; Iván Javier Movilla-de-Oro; María-del-Carmen Jiménez-Barriosnuevo

lo que se ven fortalecidas las capacidades neuro-cognitivas, la creatividad e incluso las habilidades sociales como la responsabilidad social, el trabajo en equipo.

En lo que concierne al desarrollo de habilidades físicas, se pueden aprovechar residuos de larga vida como los plásticos para para crear distintos juegos e instrumentos didácticos alternativos como conos, pinos, ula-ula, raquetas, pelotas, elásticos para trabajar la fuerza, entre otros (Cano, 2015). Es decir, se puede poner en práctica el uso de estos residuos como opción para elaborar e implementar juegos didácticos con implementos elaborados a partir de la reutilización de residuos sólidos, esto con la finalidad de servir de apoyo al momento de realizar un deporte o generar alternativas ecológicas en el campo de prácticas deportivas.

La reutilización artística de desechos sólidos consiste en hacer obras de arte con objetos que la mayoría de las personas consideran basura (Montiel, 2012), específicamente se utilizan elementos que, a simple vista, no tienen una vida útil, pero, se pueden convertir en una pieza fundamental de dichas obras y, en algunos casos, pueden hasta generar retribuciones económicas. Dentro de este proceso de reutilización artística destaca el upcycled art (en inglés) o arte de residuos reutilizados o reciclados, este tipo de arte busca la transformación de residuos como papel, cartón, madera, cristal, plástico, metales, caucho, entre otros, que normalmente serían desechados, para darle un uso artístico creando obras de arte que demuestran belleza y estética, superando el valor económico, cultural y social del producto original (Línea Verde, 2017).

\section{CONCLUSIONES}

Con el estudio se buscó abordar el principio de la transversalidad curricular como enfoque para la generación de una CA, enmarcada en la reutilización de los residuos sólidos desde las áreas de educación física y artística, todo en el contexto de la EA. Pues, entre los múltiples factores que hoy día impactan el medio ambiente, la generación acrecentada de residuos sólido en la globalidad del planeta, ofrece un horizonte para nada alentador 


\section{CIENCIAMATRIA}

Revista Interdisciplinaria de Humanidades, Educación, Ciencia y Tecnología

Año VII. Vol. VII. N¹. Edición Especial. 2021

Hecho el depósito de ley: pp201602FA4721

ISSN-L: 2542-3029; ISSN: 2610-802X

Universidad Nacional Experimental Francisco de Miranda (UNEFM). Santa Ana de Coro. Venezuela

Mario Sahamir Montes-Pacheco; Iván Javier Movilla-de-Oro; María-del-Carmen Jiménez-Barriosnuevo

en los años venideros, dado el evidente deterioro de los distintos entornos y ecosistemas. En virtud de la revisión teórica realizada se pueden exponer los siguientes señalamientos: La construcción de una sociedad inmersa en el DS para la superación y/o minimización de los problemas medioambientales, solo se puede lograr suscitando una CA basada en la idea de que el mejoramiento de la calidad de vida de la especie humana pasa porque la actividad económica se despliegue de forma amigable con el sistema natural. Es decir, se asuma axioma fundamental el desarrollo de lo económico, lo social y lo medioambiental como sistema armónico.

Para ello es primordial un cambio en los modelos de paradigmas que rigen las formas de vida, la construcción de conocimientos y las políticas que regulan los procesos de atención y protección del medio ambiente. Siendo aquí, donde tiene asidero la EA como proceso epistémico, ético-axiológico y político para la creación de conciencia planetaria, a partir de la comprensión y la acción transformadora de la realidad del entorno socioambiental.

La EA como política educativa que busca el desarrollo de conocimientos y saberes, enmarcados en el respeto y la responsabilidad en cuanto al cuidado de los entornos para la sostenibilidad ambiental, representa un instrumento para el cambio y la trasformación humana. Asimismo, es un proceso que, al permear transversalmente todo el currículo educativo, se constituye en estrategia interdisciplinaria, que permite abordar, desde múltiples perspectivas y visiones del conocimiento, la compleja realidad socioambiental haciéndola formativa.

En este contexto de la EA para la sostenibilidad de los entornos, la reutilización de los RS en las instituciones educativas se presenta como estrategia pedagógica-didáctica que posibilita la construcción de espacios de aprendizajes que coadyuvan a una cultura ecológica en pro del uso racional de los recursos, la protección y el cuidado del medio ambiente, mediante el despliegue de proyectos escolares, como los PRAE, para la recuperación y transformación de diferentes materiales de RS. 
Asimismo, estos proyectos pueden y deben ser asumidos con visión transversal. Por consiguiente, la Educación Física y la Educación Artística son espacios de aprendizaje propicios para la construcción de conocimientos y saberes interdisciplinarios y contextualizados a la realidad social que se hace educativa.

\section{REFERENCIAS}

Alaña, T., Capa, I. y Sotomayor, J. (2017). Desarrollo Sostenible y evolución de la legislación ambiental en las MIPYMES del Ecuador. Universidad y Sociedad, 9(1), 91-99. Recuperado de http://rus.ucf.edu.cu/

Aparicio, J., y Rodríguez, C. (2019). Transversalización curricular ambiental en educación superior mediante comités de diseño curricular. RIDE, 10(19), e018. DOI https://doi.org/10.23913/ride.v10i19.524

Aparicio, J., Rodríguez, C., Beltrán, J. y Sampedro, L. (2014). Transversalidad del eje medio ambiente en Educación Superior. Revista Iberoamericana de Ciencias, 1(1), 163-172. Recuperado de http://www.reibci.org/publicados/2014/mayo/4568505.pdf

Ardila, S., Vergara, Y. y González, M. (2018). La Educación Ambiental en el Marco del Plan Estratégico Territorial PET (2012-2018) de la Comuna 1 de Soacha. Una Propuesta para Contribuir al Desarrollo Local. (Tesis de postgrado). Universidad Distrital Francisco José de Caldas, Bogotá, Colombia. Recuperado de https://repository.udistrital.edu.co/handle/11349/15343

Área Metropolitana del Valle de Aburrá (2008). Guía para el Manejo Integral de Residuos. Recuperado de https://n9.cl/ufnd9

Argueta, M. (2009). Ejes transversales en el currículo de la formación inicial de docentes. Recuperado de https://n9.cl/5xsdd

Asamblea General de las Naciones Unidas (2010). Desarrollo Sostenible. Recuperado de https://n9.cl/pvxz

Asamblea General de las Naciones Unidas (1987). Informe de la Comisión Mundial sobre el Medio Ambiente y el Desarrollo. Recuperado de https://n9.cl/ro3 
Avendaño, W. (2012). La Educación Ambiental (EA) como herramienta de la Responsabilidad Social (RS). Revista Luna Azul, 35, 94-115. Recuperado de https://www.redalyc.org/pdf/3217/321727349006.pdf

Banco Mundial (2018). Los desechos 2.0: Un panorama mundial de la gestión de desechos sólidos hasta 2050. Recuperado de https://n9.cl/mnh3u

Bayón, P. (2006). Educación Ambiental, Participación y Transformación Social Sostenible en Cuba. Interface, 3(3), 89-104.

Calderón, S. y Caicedo, C. (2019). Educación Ambiental: Aspectos relevantes de sus antecedentes y conceptos. Revista Ingeniería y Región, 22, 14-27. DOI https://doi.org/10.25054/22161325.2565

Cano, L. (2015). Elaboración de material didáctico para las clases de Educación Física, a partir de la utilización de elementos reciclables como estrategia de innovación pedagógica y de conservación del medio ambiente. (Tesis de pregrado). Universidad Pedagógica Nacional Centro Valle de Tenza. Sutenza, Colombia. Recuperado de https://n9.cl/0cwb9

Casaña, S. (2017). Educación ambiental desde una perspectiva interdisciplinaria. Revista Vinculando. Recuperado de https://n9.cl/jlwse

Choles, V. (2013). Gestión Integral de Residuos Sólidos en colegios sostenibles: modelos y tendencias. (Tesis de pregrado). Pontificia Universidad Javeriana. Bogotá, Colombia. Recuperado de https://n9.cl/hllyi

CEUPE (s/f). ¿Cómo impacta la humanidad en el medio ambiente? CEUPE Magazine. Recuperado de https://n9.cl/m7j9g

Colombia Aprende (2020). Desarrollo Sostenible. Recuperado de https://n9.cl/vbsyn

Departamento Administrativo Nacional de Estadística (2018). Boletín Técnico: Cuenta ambiental y económica de flujos de materiales - residuos sólidos (2017-2018). Recuperado de https://n9.cl/5tabe

Educar para el Desarrollo Sostenible. (agosto - septiembre de 2005). Altablero. Recuperado de https://n9.cl/7vat 
Espinoza, G. (2007). Gestión y Fundamentos de Evaluación de Impacto Ambiental. Recuperado de https://n9.cl/ikwii

Esquivel, E., Cifuentes, L., Suarez, M. y Delgado, M. (2018). Educación ambiental sostenible a través de la responsabilidad social. (Tesis de maestría). Recuperado de https://n9.cl/igwzt

Ezquerra, V. (2020). Como lograr una sociedad sostenible. Recuperado de https://n9.cl/zm9i3

Ferrini, R. (1997). La transversalidad del currículo. Revista Electrónica Sinéctica, 11, 1-9. Recuperado de https://n9.cl/i4lpk

Flores, R. (2012). Investigación en educación ambiental. Revista mexicana de investigación educativa, 17(55), 1019-1033. Recuperado en 16 de octubre de 2021, de https://n9.cl/ingmp

Fuentes, L., Caldera, Y. y Mendoza, I. (2006). La transversalidad curricular y la enseñanza de la educación ambiental. Orbis, 2(4), 39-59. Recuperado de https://www.redalyc.org/pdf/709/70920403.pdf

García, O. (2015). La relación entre educación ambiental y cultura ambienta. Roca, 2(11), 77-86.

Recuperado

de https://revistas.udg.co.cu/index.php/roca/article/view/1620/2883

Garrido, F. (2016). Reutilización de Residuos Sólidos como Alternativa de Formación en la Conservación del Ambiente Elaborando Nuevos Materiales para el Docente de Educación Inicial. Revista Scientific. 1(1), p. 169-189. Recuperado de https://www.redalyc.org/journal/5636/563660226011/html/

Henríquez, C. y Reyes, J. (2008). La Transversalidad: un reto para la educación primaria y secundaria. Recuperado de https://n9.cl/ytpgc

Lévi-Strauss, C. (1974). Antropología Estructural. Recuperado de https://n9.cl/mub3i

Ley 99 (N. 41.146) (1993). Diario Oficial, 22 de diciembre de 1993. Recuperado de https://n9.cl/4j02r 
Ley 115 (N. 41.214) (1994). Diario Oficial, 08 de febrero de 1994. Recuperado de https://n9.cl/95es

Línea Verde. (2017). Día mundial del reciclaje. Ceuta, España.: Línea Verde Smart City. Recuperado de https://n9.cl/d4ij3

Martínez, R. (2010). La importancia de la educación ambiental ante la problemática actual. Revista Electrónica Educare, 14(1), 97-111. Recuperado de https://www.redalyc.org/pdf/1941/194114419010.pdf

MAVDT-MEN, (2003). La Educación Ambiental: Política Nacional. Recuperado de https://n9.cl/lod4h

Mera C., Barreiro, A., Bermúdez, R., Cedeño, B., y Vera, D. (2020). La revisión sistemática de literatura utilizando recursos electrónicos en el desarrollo de proyectos: revisión sistemática de literatura en el desarrollo de proyectos. UNESUM-Ciencias, 4(2), 1-11. $\quad$ https://doi.org/10.47230/unesumciencias.v4.n2.2020.220

Montiel, K. (2012). Reciclaje artístico: Transformación de Desechos. UDLAP [Mensaje en Blog]. Recuperado de https://n9.cl/m0oej

Morín, E. (2004). Tierra Patria. Recuperado de https://n9.cl/5xpej

Paz, L., Avendaño, W. y Parada, A. (2014). Desarrollo conceptual de la Educación Ambiental en el contexto colombiano. Luna Azul, 39, 250-270. Recuperado de https://n9.cl/1asof

Pesantez, M. (2014). Incidencia de la Cultura y Educación Ambiental en los Estudiantes del Colegio Dr. José María Velasco Ibarra del Cantón El Guabo con Respecto a la Protección y Conservación de la Naturaleza Periodo Lectivo 2013-2014. (Tesis de pregrado). Universidad Técnica de Machala. Machala, El Oro, Ecuador, Recuperado de https://n9.cl/ne450

Red de Desarrollo Sostenible de Colombia (s/f). Gestión Ambiental. Recuperado de https://n9.cl/241kd 


\section{CIENCIAMATRIA}

Revista Interdisciplinaria de Humanidades, Educación, Ciencia y Tecnología

Año VII. Vol. VII. N¹. Edición Especial. 2021

Hecho el depósito de ley: pp201602FA4721

ISSN-L: 2542-3029; ISSN: 2610-802X

Universidad Nacional Experimental Francisco de Miranda (UNEFM). Santa Ana de Coro. Venezuela

Mario Sahamir Montes-Pacheco; Iván Javier Movilla-de-Oro; María-del-Carmen Jiménez-Barriosnuevo

Restrepo, L. y Restrepo M. (2017). Proyectos Ambientales Escolares: una alternativa para la Educación Ambiental. Biocenosis, 31(1-2), 18-22. Recuperado de https://n9.cl/075c7

Romero, I. (2017). Análisis del manejo de los residuos sólidos en instituciones educativas: estudio de caso Gimnasio Cerromar en el Municipio de Riohacha Departamento de La Guajira. (Tesis de maestría). Universidad de Manizales, Manizales, Colombia. Recuperado de https://n9.cl/2xi76

Saldaña, C. y Mesina, S. (2014). Cultura Ambiental. Recuperado de https://n9.cl/h7cbi

Sánchez, A. (2011). Manual de redacción académica e investigativa: cómo escribir, evaluar y publicar artículos. Recuperado de https://n9.cl/tls1v

Severiche, C., Gómez, E. y Jaimes, J. (2016). La educación ambiental como base cultural y estrategia para el desarrollo sostenible. Telos, 18(2), 266-281. Recuperado de https://www.redalyc.org/pdf/993/99345727007.pdf

Transversalidad. (s/f). En Diccionario de Etimología DeChile.net (2001-2021). Recuperado de https://n9.cl/mrxoz

Universidad de Nuevo León, (s/f) ¿Qué es el desarrollo sustentable? Recuperado de https://n9.cl/g508x

C2021 por los autores. Este artículo es de acceso abierto y distribuido según los términos y condiciones de la licencia Creative Commons Atribución-NoComercial-Compartirlgual 4.0 Internacional (CC BY-NC-SA 4.0)

(https://creativecommons.org/licenses/by-nc-sa/4.0/). 